\title{
Multi-agent Modeling Simulation of In-vitro T-cells for Immunologic Alternatives to Cancer Treatment
}

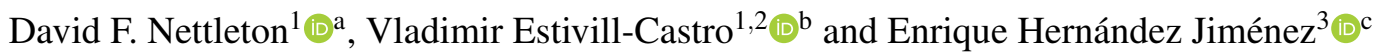 \\ ${ }^{1}$ Department of Information and Communications Technology (DTIC), Universitat Pompeu Fabra, Spain \\ ${ }^{2}$ School of ICT, Griffith University, Brisbane, Australia \\ ${ }^{3}$ Institut d'Investigació Biomèdica de Bellvitge, Barcelona, Spain
}

Keywords: Simulation, Multi-agent Systems, Immunology, Cancer Cells.

Abstract: There is exciting news in recent developments suggesting the potential to treat some human cancers by stimulating the patients own immune system. However, there is still much to understand; therefore, modelling the battle between those cells that are constituents of the human immune system against tumorous cells can significantly provide insights as mathematical modelling has done regarding the immune system behaviour against virus infections. In this paper we innovate in two directions. First, we move the modelling of immune struggles from the sphere of ordinary-differential equation models to the modelling by multi-agent simulations. We highlight the advantages of the multi-agent simulation, for example the consideration of elaborate spatial proximity interactions. Secondly, we move away from the realm of infectious diseases to the complex modelling of the stimulation of T-cells and their participation in fighting cancerous cell tumours.

\section{INTRODUCTION}

Recent developments in immunotherapy (ElSayed, 2018; Sun, 2017) indicate remarkable progress for the treatment of some human cancers by stimulating the patient's immune system (Chen and Mellman, 2013; Fernández et al., 2013; Pardoll, 2012; Farag et al., 2002). Nevertheless, the high complexity of the human immune system remains a challenge. We require progressively more advanced and high fidelity models for understanding the immune system dynamics and mechanisms. In particular, modelling the battle between those cells that are constituents of the human immune system against tumorous cells can significantly provide insights as mathematical modelling has already done regarding the immune system behaviour against virus infections (Wodarz, 2006). We open the field in two directions. First, we move the modelling of immune struggles from the realm of ordinary-differential equation models (Álvarez et al., 2017) to the modelling by multi-agent simulations (Bădică et al., 2018; Karsai et al., 2016; Zhao and Jin, 2005). We highlight that

\footnotetext{
a (iD https://orcid.org/0000-0002-5852-7716

b (i) https://orcid.org/0000-0001-7775-0780

c (iD) https://orcid.org/0000-0002-8232-8151
}

the multi-agent simulation enables consideration of elaborate spatial proximity interactions. Such interactions are extremely important in the modelling of competition and predator-prey scenarios in ecological systems (Karsai et al., 2016). Secondly, we move from the realm of infectious diseases to the complex modelling of the stimulation of T-cells and their participation in fighting cancerous cell tumours. Most cancers are not caused by transmissible pathogens; but, cancer immunotherapy suggests treatment analogous to infections (Chen and Mellman, 2013).

Adopting a system approach and modelling the dynamics analogously to ecological systems (Wodarz, 2006) has enabled tremendous progress in the understanding of the interactions between pathogens and the immune system. Such dynamics is modelled by increasingly more complicated systems of ordinary differential equations that abstract elements of what is known by experimental means (Álvarez et al., 2017; Eftimie et al., 2016; Linderman et al., 2010).

The mathematical models of infections capture population dynamic interactions of two types (Ada, 1997; Wodarz, 2006). One is predator-prey interactions, where predators kill prey, and the predator population grows until much fewer prey results also in a decrease of predator numbers. This is usually the scenario of killer cells responding to a viral infec- 
tion. Secondly, competition models abstract scenarios where two species dispute a common resource (a source of food). In the case of the immune system, one immune cell type may expand more rapidly upon exposure to infection, preventing another type of immune cell from growing its numbers.

Such mathematical modelling stimulates the experimental agenda. In the realm of viral infections, different models of how CTL (cytotoxic T lymphocytes or killer $\mathrm{T}$ cells) are assisted by CD4 $\mathrm{T}$ cell ( $\mathrm{T}$ cells that bear the CD4 marker of helper cells) in the primary response and the resolution of an infection as well as the memory phase prompted many experiments (Wodarz, 2006, Chapter 4). In particular, immunological memory is the preservation of a much larger number of specific CTL cells after a virus infection than before the infection. This memory capacity of the immune systems has delicate interactions with the eradication of the infection. The mathematical models suggest that there is a trade-off. The virus population is reduced by a high responsiveness and a long life span of the memory CTL (Wodarz, 2006).

However, while there has been an extensive literature of the application of dynamic systems models first to ecological systems (Chase et al., 2002; Kingsland, 2015) and then to the immune systems (Wodarz, 2006), there is a recent opportunity in two directions. First, to use the technology of multi-agent simulation and second to apply it to the emerging promise of the immune systems potential to fight cancer tumours (Chen and Mellman, 2013). Therefore, in this paper, we describe and test a modelling technique based on multi-agent systems for simulating systemic and inter-cellular behaviour for NK (natural killer) and cancer cells. The clinical approach is to potentiate the body's own immune system, using reprogrammed natural killers (NK is a type of lymphocyte; that is a white-blood cell) to attack the cancer (or tumour) cells. Therefore, no harm is caused to normal cells, in contrast to widely used chemo-therapy approaches.

In order to be effective, NK cells require an optimum programming against the particular cancer cell adversary. This requires an individualized study of the patient followed with a directed trial and error process which typically is tested on mice with a 14-day evolution period. Computer simulations could run in just a few minutes, with a much lower economic cost and could approach a realistic simulation performance relative to the mouse and in-vitro experimentation. Thus, multi-agent simulation could provide an important step forward in accelerating the fight against cancer-cell growth.

We incorporate medical know-how to build a multi-agent simulation model with some degree of realism. Every model is an abstraction, but we will highlight the aspects that a multi-agent simulation offers over the dynamic systems approach of using sets of differential equations. We benchmark our simulations against state of the art empirical in-vitro clinical experiments, as described by Fernández et al. (2013). Our results demonstrate that an agent system can accurately model the in-vitro experiments in terms of cytotoxicity, but without the need for defining a set of differential equations as in dynamic system models nor a priori assumptions relative to the system, and giving greater flexibility for trying many different scenarios. Our implementation uses random interaction and cellular random movement in space.

\section{THE CASE FOR MULTI-AGENT SIMULATIONS}

Purely clinical approaches to immunological treatment of cancer rely heavily on the use of in vitro trials with live mice requiring highly specialized parameterisations. Such parameterisations require deep medical knowledge. A significant part of the challenge is to anticipate behaviour, and as we explained before, so far in this field, the research has focused on mathematical modelling in the realm of dynamic systems abstracted as systems of differential equations (Álvarez et al., 2017).

But, the techniques to formulate models of biological systems, and to solve them includes also agent-based models, stochastic modelling techniques, differential equations, spatial simulations, and direct application of Gillespie's stochastic simulation algorithm (Barnes and Chu, 2015). Differential equations are suitable abstractions at the macro-level, but multi-agent systems are suitable models at the microlevel behaviour that can determine the macro-level behaviour. Sets of ordinary differential equations (ODEs) have limitations pertaining problems involving spatial interactions or emerging properties (Borshchev and Filippov, 2004). Borshchev and Filippov (2004) and others (Figueredo et al., 2014) argue ODE methods exclude emergent and stochastic behaviour.

Louzoun (2007) consider ODE methods too narrow, using few reactive types focused on simple dynamics. Louzoun (2007) argues that immunological modelling should incorporate genomic data and exploit computing power. His review (Louzoun, 2007) suggests that applications of mathematical models in immunology are shifting to immunomics: the concepts of high-throughput measurements and system immunology. Models of ODE systems for immunology are categorised as deterministic compartmental 
models (Figueredo et al., 2014) because continuous values are used for all aspects of the modelling of individuals in the environment; while multi-agent systems explicitly represent the individuals. Stracquadanio et al. (2011) argue that agent-based models "seem to be the best suited abstraction to handle the great complexity of the immune system". We acknowledge that the discrete-event scheduling of Gillespie methods has been incorporated with multiagent simulation into mixed platforms (Montagna et al., 2016). In particular, some variants of Gillespie's discrete-event scheduling have been applied to the simulation of the spatial interaction between cells (Shimoni et al., 2011; Engblom, 2019).

The model accuracy is believed to be negatively impacted by the exclusion of information on proximity (Stracquadanio et al., 2011) because most immune interactions take place within local spatial regions of the environment.

This advantage of multi-agent systems has been illustrated in the field of ecology (Bădică et al., 2018) and other specific disciplines (Montagna and Viroli, 2010; Montagna et al., 2010, 2016). Multi-agent systems have been used to explore what-if scenarios of environments that combine cell proliferation and random walks of cells on regular lattices, reactions for nutrients and growth factors (Figueredo et al., 2013a).

Researchers use different modelling environments, but for multi-agent system modelling, the Repast Simphony (North et al., 2013) is common. In particular, Repast Simphony was used to illustrate multi-agent systems simulations as an alternative to dynamic systems of differential equations for the classical predator-prey scenario (Tatara et al., 2006).

\section{RELATED WORK}

The nearest work to our approach here is the application of agent simulations of immune cell (effectors) and tumour cell interactions (Figueredo et al., 2013b, 2014). The focus of Figueredo et al. (2013b) and Figueredo et al. (2014) is to compare a differential equation model (Gillespie) with an agent based model. Figueredo et al. (2013b) and Figueredo et al. (2014) find that the agent-based model effectively simulates the system and also find patterns which were unseen by the former. However, they do not use distance based parameters, and as mentioned earlier, spatial information (proximity) is considered crucial (Stracquadanio et al., 2011). We do incorporate proximity in our model. Figueredo et al. (2013b) and Figueredo et al. (2014) do not relate the effector and tumour cells to real biological equivalents; their analysis is in-silico (Figueredo et al., 2014); that is, pure simulation. Thus, Figueredo et al. (2013b) and Figueredo et al. (2014) simulations were not matched against any real experimental values. However, they did include parameters for cell life and damage incurred to the effector cells by the tumour cells. In terms of physical characteristics, IL2 is stimulatory for the effector cells and TGF-beta suppresses the effectors and stimulates tumour growth.

An advantages of multi-agent systems is the discrete event integration of the engagement within the agents in a spatial sphere of influence. Other related work incorporating spatial considerations into an ecological model is in the area of computational epidemiology (Jindal and Rao, 2017).

\section{SIMULATION CASE STUDY}

Firstly, we will define the following acronyms: IL15 (Interleukin-15), NKG2D (Natural Killer-gene 2D), MICA (MHC class I polypeptide-related sequence A), ULBP2 (UL16 binding protein 2) and HLA class 1 (Human Leukocyte Antigen).

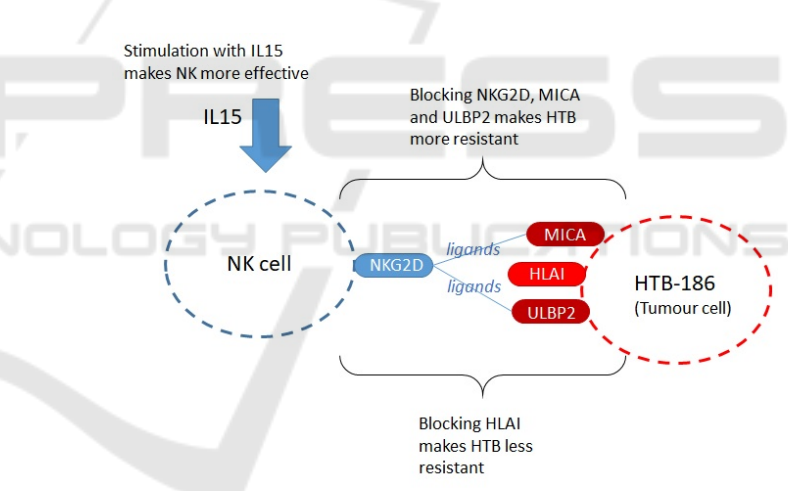

Figure 1: Features on NK and Tumour cells (HTB-186).

We chose as our case study study the interaction between tumour cells (HTB-186) and NK cells under different conditions (Fernández et al., 2013). Fernández et al. (2013) cultivated NK and HTB-186 tumour cells in vitro from 54 medulloblastoma patients. For the simulation we focus on the results shown in (Fernández et al., 2013, Fig. 3), which record the resulting cytotoxicity of NKs for different blocking and stimulation conditions. Five conditions are recorded: (i) Resting NK; (ii) IL15 stimulated NK; (iii) Blocking of NKG2D (on NK), MICA and ULBP2 (on HTB-186); (iv) HLA class 1 blocking on HTB-186; (v) IL15 stimulated NK and HLA class 1 blocking on HTB-186. Note that (ii), (iv) and (v) favour greater cytotoxicity of NK, whereas (iii) reduces cytotoxicity of NK. In the case of (iii), this is 
because NKG2D is the NK cell receptor and MICA and ULBP2 are tumour (HTB-186) ligands for NK. Refer to Figure 1 for an illustration of features on the $\mathrm{NK}$ and tumour cells. Also note that, as the in-vitro experiments have a duration of approximately three hours, we do not consider proliferation or cell death.

\subsection{Representation as a Dynamic System Model}

We now illustrate how the in-vitro experiment of our case study ( Fernández et al. (2013)) would be modelled as a "dynamic system model".

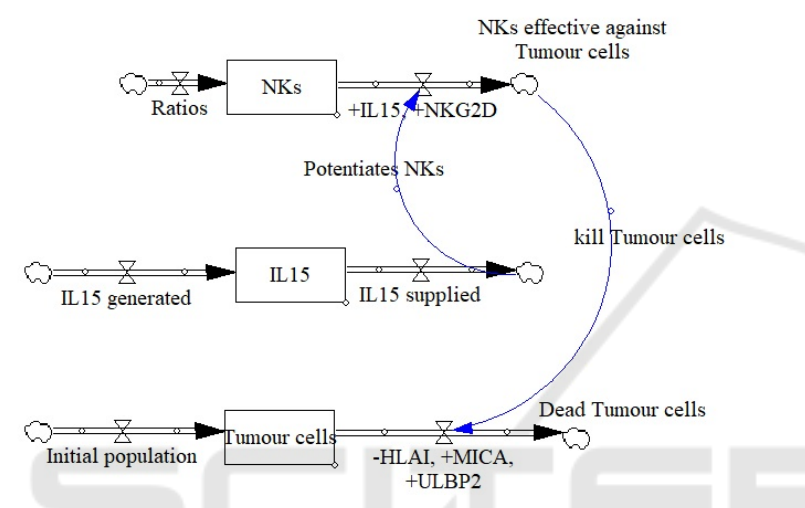

Figure 2: Stock and flow diagram for NK and HTB-186 tumour.

A dynamic system model typically consists of a stock and flow diagram (Figure 2), a set of differential equations to represent the behaviour of the stocks (Figure 3), a set of algebraic equations to define the flows (Figure 4), and a set of control parameters used by the system (Figure 5). In Figure 2, the three stocks are NKs, IL15 and Tumour cells. The flow on the top right indicates the number of NK cells which are effective against the Tumour cells, and this is affected positively by IL15 and the NKG2D activating receptor (hence they are preceded by $\mathrm{a}+$ ). The flow on the bottom right indicates the number of Tumour cells being killed by NKs, and this is positively affected by MICA and ULDP2 ligands (+) and negatively by HLA class I expression (-).

IL-15 is used as potential therapy to enhance NK anti-tumoral activity. Additionally, IL-15 has been shown to enhance the anti-tumor immunity of CD8+ $\mathrm{T}$ cells in pre-clinical models. In the current model IL-15 is considered as a stimulator, but without localization in the physical space, assuming it is homogeneously distributed and affects all NK's equally. In this sense, it's considered in the same way as MICA, NKG2D and ULBP. They are either present (or ac- tive) or absent (or blocked). This also agrees with the predator-prey model: NK are predators, HLA are the prey, and IL-15 acts as a stimulator for NK. However, in future work the definition of IL-15 as an agent could be considered.

\begin{tabular}{|c|c|c|c|}
\hline $\begin{array}{l}\text { Equation } \\
\text { no. }\end{array}$ & Stock & Equation & Units \\
\hline 1 & NKs & $\frac{d N K(t)}{d t}=-E f f e c t i v e N K s(t)$ & NK \\
\hline 2 & $\begin{array}{l}\text { Tumour } \\
\text { cells }\end{array}$ & $\frac{d \text { Tumour cells }(t)}{d t}=-$ Dead Tumour cells $(t)$ & $\begin{array}{c}\text { Tumour } \\
\text { cells }\end{array}$ \\
\hline 3 & IL15 & $\frac{d I L 15(t)}{d t}=-I L 15$ supplied $(t)$ & volume \\
\hline
\end{tabular}

Figure 3: Differential equations for stocks in Figure 2.

\begin{tabular}{|c|c|c|c|}
\hline $\begin{array}{c}\text { Equation } \\
\text { no. }\end{array}$ & Flow & Equation & Units \\
\hline 1 & Ratio of NKs & Ratio of $\mathrm{NKs}(\mathrm{t})=\mathrm{NK}$ base population $(\mathrm{t}) \times$ Ratio & \# NK cells \\
\hline 2 & Effective NKs & Effective NKs(t) $=N K s(t)$. IL15(t). NKG2D(t) & NK per hour \\
\hline 3 & IL15 generated & IL15 generated(t) & $\begin{array}{l}\text { Nanogram per } \\
\text { millilitre per hour }\end{array}$ \\
\hline 4 & |L15 supplied & IL15 supplied(t) & $\begin{array}{l}\text { Nanogram per } \\
\text { millilitre per hour }\end{array}$ \\
\hline & $\begin{array}{c}\text { Initial } \\
\text { population } \\
\text { tumour cells }\end{array}$ & Tumour cell base population(t) & \# Tumour cells \\
\hline 6 & $\begin{array}{l}\text { Dead tumour } \\
\text { cells }\end{array}$ & $\begin{array}{l}\text { Dead tumour cells }(t)=\text { Effective } N K(t) \cdot-\text { HLA } \\
\text { Class1 }(t) \cdot \text { - MICA(t) - ULBP2(t) }\end{array}$ & $\begin{array}{l}\text { \# Dead tumour } \\
\text { cells }\end{array}$ \\
\hline
\end{tabular}

Figure 4: Differential equations for flows of Figure 2.

\begin{tabular}{|cccc}
\hline N & Parameter & Value & Units \\
\hline 1 & MICA threshold & 50 & Positive cells n(\%) \\
\hline 2 & ULBP2 threshold & 25 & Positive cells n(\%) \\
\hline 3 & IL-15 concentration & 25 & $\mathrm{Ng} / \mathrm{ml}$ \\
\hline 4 & $\begin{array}{c}\text { Mouse anti-ULPB2 } \\
\text { monoclonal IgG2A antibody }\end{array}$ & 2,37 & Hours, 으 \\
\hline 5 & NKG2D IgG1 antibody & 30 & Minutes \\
\hline 6 & $\quad$ Ratio & $1,2,4,8$ & Units \\
\hline 7 & $\begin{array}{c}\text { Initial NK cell population } \\
8\end{array}$ & 400 & \#cells \\
\hline & $\begin{array}{c}\text { Initial Tumour cell } \\
\text { population }\end{array}$ & 400 & \#cells \\
\hline
\end{tabular}

Figure 5: Control parameters for the dynamic system of Figure 2.

\section{AGENT MODEL}

With reference to the simulation case study described in Section 4, we will simulate two agent classes (using Repast Simphony (North et al., 2013)): agent-type NK and agent-type Tumour. In our spatial environment, both classes are reactive agents (acting independently, exhibiting control over their internal state). Such individual agent behaviour is defined by global rules as well as local rules.

NK-cells have short-term goal directed behaviour for probabilistically aiming for a Tumour cell. We 
Table 1: Agent control parameters.

\begin{tabular}{||c|c||c|c|c|c|c|c||}
\hline \hline \multicolumn{2}{|c||}{ Tumour cells } & \multicolumn{9}{c|}{ NK-cells } \\
\hline $\begin{array}{c}\text { speed of } \\
\text { movement }\end{array}$ & $\begin{array}{c}\text { reproduction } \\
\text { rate }\end{array}$ & $\begin{array}{c}\text { speed of } \\
\text { movement }\end{array}$ & $\begin{array}{c}\text { reproduction } \\
\text { rate }\end{array}$ & $\begin{array}{c}\text { range of } \\
\text { kill distance }\end{array}$ & $\begin{array}{c}\text { range of distance } \\
\text { detection }\end{array}$ & $\begin{array}{c}\text { kill } \\
\text { probability }\end{array}$ & $\begin{array}{c}\text { dormant/activate } \\
\text { period }\end{array}$ \\
\hline 0.003 & 0.0 & 0.01 & 0.0 & 0.5 & 1.0 & 0.6 & 2 \\
\hline \hline
\end{tabular}

name Agent control parameters those values regulating the agents' actions; these control parameters specify behaviour for the agents. A second set of parameters incorporates the experimental cellular information.

The metric used to evaluate the fit of the simulation to the in-vitro experiments is the number of surviving Tumour cells when equilibrium is reached. That is, the difference between the Tumour cell count from Fernandez's in-vitro experiments (Table 4) and the Tumour cell count resulting from the agent model (Table 5), which are shown in Figure 10.

The Repast source code of the system is available at:github.com/dnettlet/AgentSim1.

In the first control parameter set (refer to Table 1), for each agent type (NK or Tumour) we incorporate the following control parameters (and their values) based on experimental information ( Fernández et al. (2013)): speed of movement of the agent instances in the defined space; reproduction rate of the agents; distance at which a given agent type NK is able to detect another agent type Tumour, and the distance at which NK can kill (remove from participation in the simulation) an agent of the class Tumour; and the probability that, when NK is within distance to kill a Tumour agent, it effectively eliminates the Tumour agent. The dormant period is the number of cycles an NK-cell requires to become dormant in the absence (within detect distance) of Tumour cells. Likewise, the active period is the number of cycles an NK-cell requires to become active in the presence (within detect distance) of Tumour cells.

Table 1 shows the initial value settings for the control parameter set. Also, we use the values by Fernández et al. (2013) to assign set-up values:

- Number of cycles without detecting tumour cells for NK cells to become dormant (assigned as 2)

- Number of cycles having detected tumour cells for NK cells to become active (assigned as 2)

- Initial number of tumour cells (assigned as 400)

- Initial number of NK cells (assigned as 400 for ratio $1: 1$ )

Each of the agent control parameters of Table 1 can be related with a real biochemical process (e.g. kill distance approximates the docking distance).

The second control parameter set (Table 2) constitutes the experimental parameters of the real in-vitro
Table 2: Experiments, and their settings.

\begin{tabular}{||c||c|c|c|c|c|c||}
\hline \hline Experiment & Resting & IL15 & ULBP2 & MICA & NKG2D & HLAI \\
\hline 1 & 1 & 0 & 0 & 0 & 0 & 0 \\
3 & 0 & 1 & 0 & 0 & 0 & 0 \\
4 & 0 & 0 & 1 & 1 & 1 & 0 \\
5 & 0 & 0 & 0 & 0 & 0 & 1 \\
\hline \hline
\end{tabular}

Table 3: Factors to incorporate stimulus or inhibition into NK-cells behaviour.

\begin{tabular}{||c||c|c|c|c|c|c||}
\hline \hline Experiment & Resting & IL15 & ULBP2 & MICA & NKG2D & HLAI \\
2 & 1.1 & 0 & 0 & 0 & 0 & 0 \\
3 & 0 & 1.1 & 0 & 0 & 0 & 0 \\
4 & 0 & 0 & 0.9 & 0.9 & 0.9 & 0 \\
5 & 0 & 0 & 0 & 0 & 0 & 1.1 \\
\hline \hline
\end{tabular}

experiments by Fernández et al. (2013). The original labels for these experiments are 1 to 5 Fernández et al. (2013). Each in-vitro experiment is defined by six test conditions.

Table 2 indicates for each experiment, whether an experimental feature is present or not. For example, in Experiment 5, IL15 and HLAI are present, indicated by a 1 . When a feature is present, it acts as a stimulant or inhibitor on the cytotoxicity (refer to Fernández et al. (2013)).

We incorporate the experimental features into the multi-agent simulation, as having either a stimulant role or inhibitor role in the agents parameters (the values in Table 1). We apply them as multipliers that either magnify or reduce the effect of the agents behavioural parameters. A magnifying multiplier will be larger than 1 and a reducing multiplier will be less than 1 . Therefore, the conditions in Table 2 were corrected to the multipliers in Table 3.

We apply this effect (which influences NK-cell behaviour) by affecting the behavioural parameters of NK-cells of Table 1. For example, the IL15 and HLAI features potentiate the cytotoxicity, (NKs kill a higher percentage of Tumour cells) and they are both present in Experiment 5, so the weights from Table 3 adjust the NK-cell agent control parameters as follows:

$$
\text { kill distance }=\prod_{i=1}^{\text {number of features }} \text { kill distance } \times w_{i j}
$$

where $j$ is fixed as the experiment number ( 5 in this case). As an example, consider for Experiment 5 (Table 3, Row 5) that the non-zero weights $w$ are 1.1 (for IL15) and 1.1 (for HLAI), and the kill distance is initially set to 0.5 . Thus, the new kill distance in the first iteration will be $0.5 \times 1.1=0.55$, and in the 
second iteration, $0.55 \times 1.1=0.605$. Similarly,

$$
\text { detect distance }=\prod_{i=1}^{\text {number of features }} \text { detect distance } \times w_{i j}
$$

On the other hand, in Experiment 3 (Table 3, Row 3) the NKG2D feature dampens the cytotoxicity (reduces the NK kill rate on Tumour cells). This is a reduction weight of 0.9 that is multiplied with the agent control parameters, so experiments with the presence of NKG2D will see both "kill distance" and "detection distance" affected by the value 0.9 .

We incorporated the effect of experimental features to spatial behavioural features of the agents only to three agent control parameters of only the NKcells. That is, we only affect the kill distance, detection distance and kill probability of NK-cells. Besides biological realism, initial simulations with our agents showed only these behavioural components had meaningful effects (number of Tumour cells remaining when system reaches equilibrium). Never-
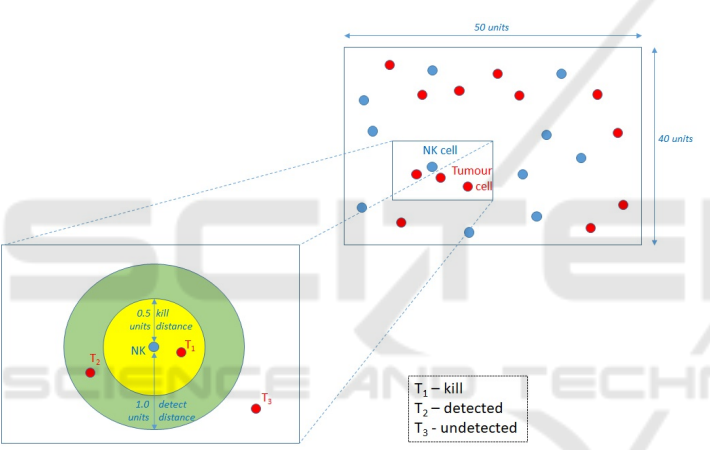

Figure 6: State space definition for agent interactions.

theless, despite the incorporation of inhibitors and stimulants to NK-cells, the interactions between NKcells and tumour cells is not magnified or reduced uniformly across all experiments. Thus, the interaction of agent control parameter values (in Table 1) feature weighting (in Table 3 ) requires further regulation. To achieve such regulation, we used a third weight $w^{\prime}$ to adjust all the NK-agents behavioural components uniformly but for each experiment and ratio. That is, the weight $w^{\prime}$ adjusts the effect of the weights of Table 3 when applied to Table 1. Table 5 shows the optimised weights $w^{\prime}$ resulting in high fidelity with simulations achieving consistently the equilibrium point to the required cytotoxicity.

For example, consider that the optimizer weight $w^{\prime}$ has been settled as the value 0.73 (see Table 5) for Experiment 5 and $\mathrm{NK}$ to tumor cell ratio equal to 1 , then for all features $i$ and Experiment $j=5$, the weights $w$ in Table 3 are updated thus:

$$
\forall i, j \in I=1: 6, J=1: 5, \quad w_{i j}=w_{i j} w^{\prime}
$$

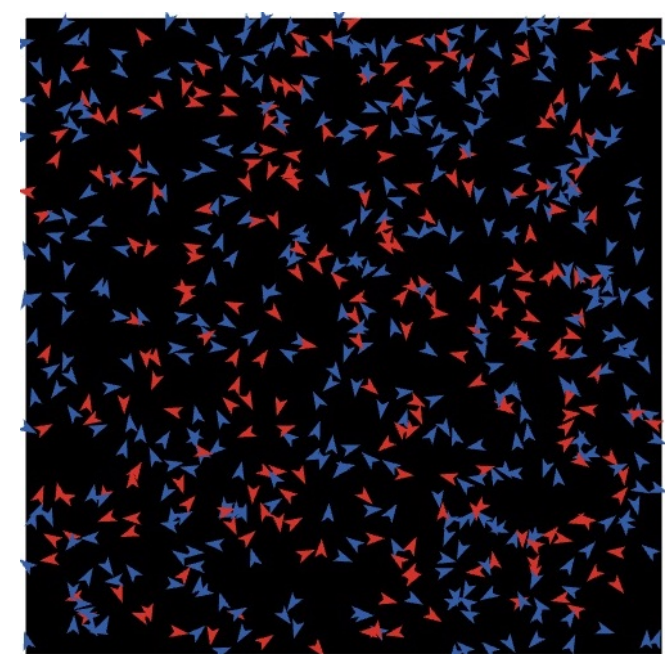

Figure 7: Agent simulation for Experiment 5, ratio 1:1.

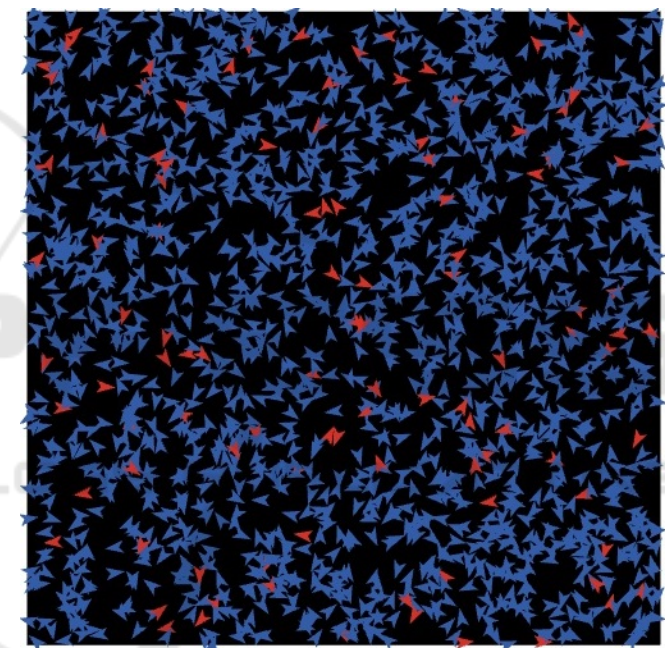

Figure 8: Agent simulation for Experiment 5, ratio 4:1.

To illustrate the process, consider for Experiment 5 (Table 3, Row 5) that the non-zero weights $w$ are 1.1 (for IL15) and 1.1 (for HLAI), and the kill distance is initially set to 0.5 . Thus, the new kill distance in the first iteration will be $0.5 \times 1.1 \times 0.73=0.4015$, and in the second iteration, $0.55 \times 1.1 \times 0.4015=0.3224$.

We repeat the experimental conditions (Table 2) for the in-vitro experiments with real NK-cells and tumour cells for four different initial ratios of NK-cells and tumour cells, following exactly the ratio of NKcells to tumour cells suggested by Fernández et al. (2013) and appearing in Table 4. Table 4 also shows survival ratios in our experiments.

Each experiment was repeated several times to verify that the same equilibrium point was reached with the same weight. In some cases the result was unstable (some runs did not stop at target) and this is 
Table 4: Resulting cytotoxicity percentage for each ratio of agent classes in in-vitro experiments.

\begin{tabular}{||c||c|c|c|c||}
\hline \hline \multicolumn{1}{|c||}{ Experiment } & \multicolumn{4}{c|}{ Ratio } \\
& 1 & 2 & 4 & 8 \\
\hline 1 & 14 & 16 & 19 & 26 \\
2 & 19 & 27 & 40 & 45 \\
3 & 10 & 10 & 11 & 13 \\
4 & 42 & 55 & 66 & 73 \\
5 & 41 & 57 & 74 & 87 \\
\hline \hline
\end{tabular}

due to the stochastic nature of the agent system. In this case, the weight value was varied slightly in upper and lower bound until a stable equilibrium was found on repeats. In future work an automatic optimization such as hill climbing with a fitness function could be used.

In summary, in our simulation, NK-cell agents and tumour-cell agents interact (refer to Figure 1) as per the effects of inhibitors and stimulants (refer to Table 3) that are tuned (by $w^{\prime}$ ) to impact the behavioural parameters (Table 1) in a two dimensional state space (as illustrated by Figure 6). The kill distance and detect distance represent the physical distance in the biological system for locking on (via ligands and receptors) and detection of chemical substances by the NK cell which signal the presence of a tumour cell. Note that the predator (NK) agents perform a random walk until a tumour cell falls within detect distance, then the NK moves directly towards the tumour.

Figure 7 and Figure 8 show two scenes from our multi-agent simulations using the Repast Simphony (North et al., 2013). Both correspond to the settings of Experiment 5, where Figure 7 has a ratio of 1:1 NK-cell agents to tumour cell agents, with initial cell populations of 400 for each agent type. On the other hand, Figure 8 is the case where the ratio is $4: 1$, with initial cell populations of $1600 \mathrm{NK}$-cell agents and 400 tumour cells. The tumour cells are coloured red and the NKs are coloured blue. For Figure 7, the system has reached an equilibrium at 237 tumour cells, which matches the cytoxicity of $41 \%$ obtained in the in-vitro experiment.

Figure 8 shows the situation where the system has reached an equilibrium at 106 tumour cells, which matches to the cytoxicity of $74 \%$ as obtained in the in-vitro experiment.

\section{EXPERIMENTS}

We now detail the results of the experiments to simulate the in-vitro clinical experiments presented by Fernández et al. (2013). Table 5 shows a total of 20 experiments given that we ran 4 ratios in 5 experiments. Our results show that for all the ratios and all of the experiments, we can successfully reproduce the cytotoxicity percentage combinations. That is, in each case, we achieve convergence to the target $\%$ of tumour cells killed with error margin (\%target vs $\%$ real) of between $-1.5 \%$ and $+2.25 \%$ (last column in Table 5). It can be seen that a lower weight (column 3) was found to be optimum for experiments 1 , 2 and 4, whereas higher values were required for experiments 3 and 5. This is due to the fact that the experimental settings involve only one inhibitor (or stimulant) in one set of experiments while the latter experiments involve more factors.

Figures 9 to Figure 11 provide graphical illustrations of the results of Table 5. In particular, Figure 9 shows the weight values $w^{\prime}$ we found for each experiment and ratio. It can be seen that for each experiment, the weights decrease inversely with respect to the ratios. Also it can be seen that higher cytotoxicity requires a higher weight but more overriding is the combination of multiple factors (especially Experiment 3). Figure 10 shows the simulated cytotoxicity for each experiment and ratio. We obtain remarkable similarity to the in-vitro results, our Figure 10 matches Fernández et al. (2013, Figure 3). Note that Figure 11 shows the error margin (real in-vitro values (Fernández et al., 2013, Figure 3) vs our multiagent simulated cytotoxicity) for each experiment and ratio. The relative errors are never more than $2.25 \%$.

Figure 12 shows a benchmark of performance of our simulations. We ran Repast Simphony 2.6 in "batch mode" for environment with different number of cells. For this benchmark, we used Experiment 5 with ratio 1:1 NK to tumour cells. We used a Lenovo laptop with Intel Core i7-7500U, CPU @ 2.7Ghz (64bit processor), 12GB RAM, Windows 1064 bit operating system. It can be seen that the processing time fits to a third degree polynomial, where 9600 cells marks the saturation point of the computer. The performance would of course be improved using a GPU and/or multi core parallel processing, which would allow for a much greater number of cells, though for the current set of experiments this was not considered necessary as the stochastic nature and distance based interactions depend on density of cells per square unit of space rather than pure quantity. However, testing with greater quantities will be a potential aspect for future work.

\subsection{Discussion}

From the results it can be seen that by manipulating the control parameters we can accurately simulate the real in-vitro experiments by Fernández et al. (2013). The flexibility of the multi-agent simulation pays-off! This promises to reduce the time needed for clinical 
Table 5: Results of Experiments 1 to 5 for different ratios of NK to Tumour cells.
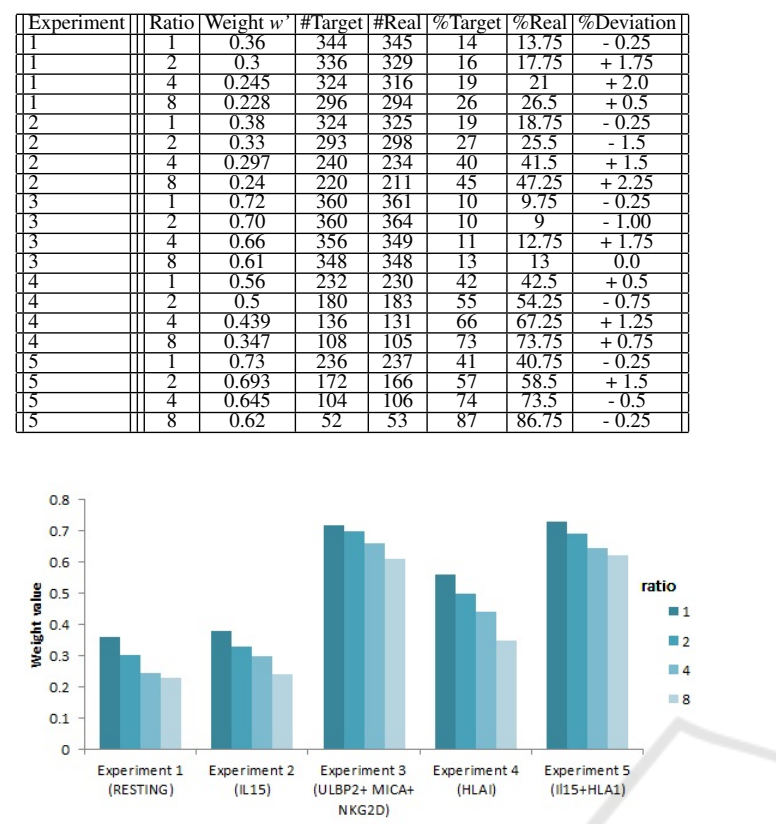

Figure 9: Weight values for each experiment and ratio.

testing for customized patient treatment where every day counts. The multi-agent system which allows the creation of thousands of 'virtual' cells permits a much closer stochastic type interaction, in contrast to the typical differential equation approach. Also, the agent simulation allows the definition of a virtual space (a grid with $N \times M$ dimensions) in which the agents move and interact. This, combined with the kill distance and detection distance parameters allow to approximate the real chemical detection between cells, and the lock-on distance between receptors and ligands. The approximation is due to the mapping onto the two dimensional grid where the agents are defined. The kill probability also reflects the stochastic/probabilistic nature of the process: the probability that an NK will realistically kill a Tumour cell when within the kill distance. Whether the NK kills a Tumour cell or not can depend on semi-random aspects such as locking orientation, particularities of the indi-

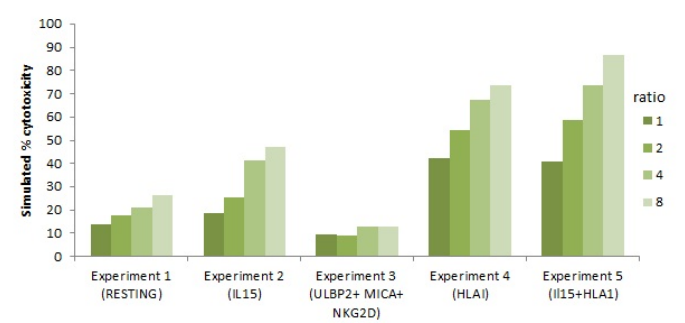

Figure 10: Simulated cytotoxicity per experiment and ratio.

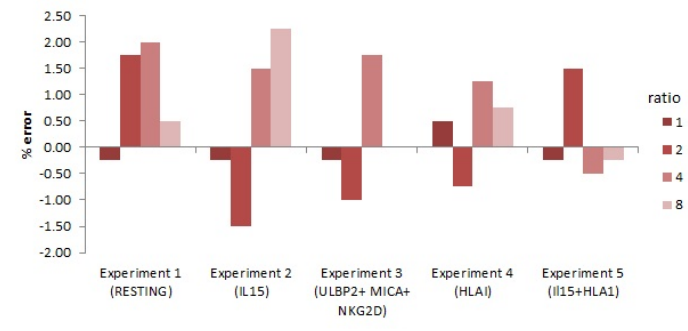

Figure 11: Error margin for each experiment and ratio.

vidual NK-cell and the Tumour cell in the encounter, and probabilistic events such as movement of adjacent cells. Finally, the steps with no tumour cells controls the predator-prey interaction, which does happen in the in-vitro environment. Here, we incorporate it as a deactivation of the NK-cell; that is, if in $N$ cycles no tumour cell has entered into the detect distance of a given NK-cell, then it becomes dormant. This parameter together with the detect distance are major parameters in steering the simulation: as more tumour cells are killed and become less prevalent, more NKcells will become inactive. However, the NK-cells reactivate again if the inverse happens: tumour cells come within detect distance during at least $N$ cycles.

For the in-vitro experiments, the activator for the NK (IL15) and the ligands/receptors (NKG2D on the NK cell, MICA, ULBP2 and HLAI on the tumour cell) were pre-selected by clinical trials, as well as the type of tumour cell (HTB-186 MB). Hence, in order to build an even more accurate decision-support system (DSS) for clinical trials, the agent simulation would need to serve as a back-end to the DSS which would, for a given combination of tumour type, NKcell type, activator and set of ligands/receptors, produce the expected result, that is the resulting kill rate (cytotoxicity). It may be infeasible to expect to build a general system for every cancer treatment, and it would be likely that specialized systems (knowledge bases) would be loaded for specific types of cancer.

One form of action could be the following: by successfully simulating a diversity of in-vitro clinical experiments, the optimum control parameters and weights would be found for the corresponding cases. This would build a knowledge-base of cases. Thus, when a new case is presented, the closest historical case would be retrieved and used as the basis for a new simulation. Ideally, it would be possible not only to find the optimum control parameter set of the agent system, but also the combination of activator, ligands/receptors to block or to potentiate maximal cytotoxicity. This would result in a system with just one parameter as input (the tumour cell type) and a set of parameters as output (immune system cell type, activators, ligands/receptors to block/potentiate, resulting 


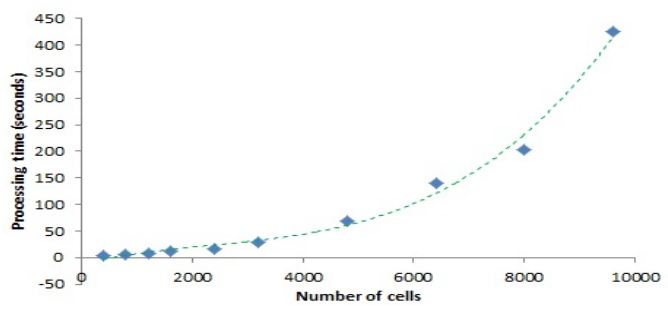

Figure 12: Number of cells vs processing time - Experiment 5 , ratio $1: 1$.

cytotoxicity). As a further enhancement, a DSS could also answer questions such as: what would be the results if we use IL2 instead of IL15? If we block ligand $B$ instead of $A$, receptor $Y$ instead of $X$, and so on. Is it possible to reach $100 \%$ cytotoxicity? In general, we could ask "what is the set of clinical parameters which maximizes cytotoxicity?"

Returning to the current simulation, there remain issues to evaluate: how close is the spatial representation to the real in-vitro physical environment. We have used a 40 by 50 unit grid, where the agents themselves have no explicit dimension, but where the detect distance value starts at 1.0 units and the kill distance starts at 0.5 units. How well does this map onto the real physical space? Also, how many cells are necessary to represent the in-vitro cell populations. We have used a minimum of 400 and a maximum of 3200 in the simulations. Is that enough to represent the stochastic behaviour? Also, the agent simulation is defined on a two dimensional grid, whereas the in-vitro is acting in a three dimensional space. These aspects limit the scope of the simulation. However, even with these limitations, we would say that the agent simulation is much more realistic than the differential equation model, and follows similar lines to agent simulations in general. As future work, we could try to implement a 3D graph-data structure instead of the 2D grid, in which graph nodes represent the cells, which are in turn, linked to neighbourhoods of adjacent cells.

Also, it could be said that the agent system per se is somewhat limited to a detect/kill functionality within a parameter-defined range. As a future step, inhibitors/stimulators could be modelled as entities instead of modulators of parameter values, to account for possible spatial effects, for example.

\section{CONCLUSIONS}

We have demonstrated the high flexibility of multiagent simulations in two aspects. First, modelling aspects of the real interaction between tumour cells and the naturally existing NK-cells, as they are inhibited or stimulated in real in-vitro environments. Secondly, we can use the multi-agent system's additional parameters to explore and optimise the best combinations which influence the inhibitors and stimulatory compounds on NK-agents to approximate the cytotoxicity. Our results show that by manipulating the control parameters we can accurately simulate the real in-vitro experiments of Fernández et al. (2013).

This is a promising step for assisting the need for clinical testing for customized patient treatment where every day counts. The multi-agent system which allows the creation of thousands of "virtual" cells permits a much closer stochastic type interaction, in contrast to the typical ODE approach.

This research has resulted in new ideas for future work, such as, how to build a true decision-support system which can give the clinical trial set-up (immune cell type, activator, which ligands and receptors to inhibit/potentiate) to maximize cytotoxicity for a given tumour cell type.

\section{ACKNOWLEDGEMENTS}

We are grateful to the anonymous reviewers for their suggestions. We are also grateful to Sergio Nettleton (Universitat Politècnica de Catalunya, Barcelona, Spain) for the Repast programming support.

\section{REFERENCES}

(1997). Adam, J. and Bellomo, N., eds., A Survey of Models for Tumor-Immune System Dynamics, Modeling and Simulation in Science, Engineering and Technology, Boston. Birkhäuser.

Álvarez, E., Toledano, V., Morilla, F., Hernández-Jiménez, E., Cubillos-Zapata, C., Varela-Serrano, A., CasasMartín, J., Avendaño Ortiz, J., Aguirre, L. A., Arnalich, F. Maroun-Eid, E., Martín-Quirós, A., Quintana Díaz, M., and López-Collazo, E. (2017). A system dynamics model to predict the human monocyte response to endotoxins. Frontiers in Immunology, 8:915.

Barnes, D. J. and Chu, D. (2015). Guide to Simulation and Modeling for Biosciences. Simulation Foundations, Methods and Applications. Springer.

Borshchev, A. and Filippov, A. (2004). From system dynamics and discrete event to practical agent based modeling: Reasons, techniques, tools. 22nd Int. Conf. of the System Dynamics Society.

Bădică, A., Bădică, C., Ivanović, M., and Dánciulescu, D. (2018). Multi-agent modelling and simulation of graph-based predator-prey dynamic systems: A BDI approach. Expert Systems, 35(5):e12263. 
Chase, J. M., Abrams, P. A., Grover, J. P., Diehl, S., Chesson, P., Holt, R. D., Richards, S. A., Nisbet, R. M., and Case, T. J. (2002). The interaction between predation and competition: a review and synthesis. Ecology Letters, 5(2):302-315.

Chen, D. S. and Mellman, I. (2013). Oncology meets immunology: The cancer-immunity cycle. Immunity, 39(1):1-10.

Eftimie, R., Gillard, J. J., and Cantrell, D. A. (2016). Mathematical models for immunology: Current state of the art and future research directions. Bulletin of Mathematical Biology, 78(10):2091-2134.

ElSayed, Z. A. (2018). Recent advances in cancer immunotherapy. QJM: An Int. Journal of Medicine, 111(suppl_1).

Engblom, S. (2019). Stochastic simulation of pattern formation in growing tissue: A multilevel approach. Bulletin of Mathematical Biology, 81(8):3010-3023.

Farag, S. S., Fehniger, T. A., Ruggeri, L., Velardi, A., and Caligiuri, M. A. (2002). Natural killer cell receptors: new biology and insights into the graft-versusleukemia effect. Blood, 100(6):1935-1947.

Fernández, L., Portugal, R., Valentín, J., Martín, R., Maxwell, H., González-Vicent, M., and PérezMartínez, A. (2013). In vitro natural killer cell immunotherapy for medulloblastoma. Frontiers in oncology, 3(94).

Figueredo, G. P., Joshi, T. V., Osborne, J. M., Byrne, H. M., and Owen, M. R. (2013a). On-lattice agent-based simulation of populations of cells within the open-source chaste framework. Interface focus, 3(2).

Figueredo, G. P., Siebers, P.-O., and Aickelin, U. (2013b). Investigating mathematical models of immuno-interactions with early-stage cancer under an agent-based modelling perspective. BMC Bioinformatics, 14(6):S6.

Figueredo, G. P., Siebers, P.-O., Owen, M. R., and Reps, J. Aickelin, U. (2014). Comparing stochastic differential equations and agent-based modelling and simulation for early-stage cancer. PLOS ONE, 9(4):e95150.

Jindal, A. and Rao, S. (2017). Agent-based modeling and simulation of mosquito-borne disease transmission. 16th Conf. on Autonomous Agents and MultiAgent Systems, AAMAS '17, pages 426-435, Richland, SC. Int. Foundation for Autonomous Agents and Multiagent Systems.

Karsai, I., Montano, E., and Schmickl, T. (2016). Bottomup ecology: an agent-based model on the interactions between competition and predation. Letters in Biomathematics, 3(1):161-180.

Kingsland, S. (2015). Alfred J. Lotka and the origins of theoretical population ecology. Proc. of the National Academy of Sciences, 112(31):9493-9495.

Linderman, J. J., Riggs, T., Pande, M., Miller, M., Marino, S., and Kirschner, D. E. (2010). Characterizing the dynamics of CID4+ T cell priming within a lymph node. The Journal of Immunology, 184(6):2873-2885.

Louzoun, Y. (2007). The evolution of mathematical immunology. Immunological reviews, 216:9-20.

Montagna, S., Donati, S., and Omicini, A. (2010).
An agent-based model for the pattern formation in drosophila melanogaster. Alife XII Conference.

Montagna, S., Omicini, A., and Pianini, D. (2016). Extending the Gillespie's stochastic simulation algorithm for integrating discrete-event and multi-agent based simulation. Multi-Agent Based Simulation XVI, volume 9568 of MABS 2015, pages 3-18, Cham. Springer.

Montagna, S. and Viroli, M. (2010). A framework for modelling and simulating networks of cells. Electronic Notes in Theoretical Computer Science, 268:115-129.

North, M. J., Collier, N. T., Ozik, J., Tatara, E. R., Macal, C. M., Bragen, M., and Sydelko, P. (2013). Complex adaptive systems modeling with Repast Simphony. Complex Adaptive Systems Modeling, 1(1):3.

Pardoll, D. M. (2012). The blockade of immune checkpoints in cancer immunotherapy. Nature reviews. Cancer, 12(4):252-264.

Shimoni, Y., Nudelman, G., Hayot, F., and Sealfon, S. (2011). Multi-scale stochastic simulation of diffusioncoupled agents and its application to cell culture simulation. PloS one, 6:e29298.

Stracquadanio, G., Umeton, R., Costanza, J., Annibali, V., Mechelli, R., Pavone, M., Zammataro, L., and Nicosia, G. (2011). Large scale agent-based modeling of the humoral and cellular immune response. Artificial Immune Systems, pages 15-29, Berlin, Heidelberg. Springer Berlin Heidelberg.

Sun, W. (2017). Recent advances in cancer immunotherapy. Journal of Hematology \& Oncology, 10(1):96.

Tatara, E., North, M. J., Howe, T. R., Collier, N. T., and Vos, J. R. (2006). An introduction to Repast modeling by using a simple predator-prey example. 2006 Conf. on Social Agents: Results and Prospects. Argonne National Laboratory.

Wodarz, D. (2006). Killer Cell Dynamics: Mathematical and Computational Approaches to Immunology. Springer, New York.

Zhao, D. and Jin, W. D. (2005). The study of cooperative behavior in predator-prey problem of multi-agent systems. Autonomous Decentralized Systems, 2005. ISADS 2005., pages 90-96. 